Original Research Paper

\title{
Data Path Management Mechanism to Mesh Base Stations in WiMAX
}

\author{
Sahar Abdul Aziz Al-Talib and Noor Raad Saadallah \\ Computer and Information Engineering Department, Electronics Engineering, Ninevah University, Mosul, Iraq
}

Article history

Received: 15-01-2019

Revised: 15-02-2019

Accepted: 21-03-2019

Corresponding Author: Sahar Abdul Aziz Al-Talib Computer and Information Department Engineering, Electronics Engineering, Ninevah University, Mosul, Iraq Email: sahar.altalib@uoninevah.edu.iq or sahar.altalib@gmail.com

\begin{abstract}
Three options are popular today for design of wireless networks Point-to-Point, Point-to-Multipoint and Mesh topologies. Worldwide interoperability of microwave access (WiMAX) is most often associated with the point-to-multipoint (PMP) topology. For some requirements, the configuration of the base stations (BSs) is aimed to be as mesh. Mesh offers a combination of point-to-point and point-to-multipoint capability by having each of the base station able to communicate with other base stations. This averts the base station element in the standard, besides the process flow across relevant interfaces. The paper proposes a Distributed Data Path Function (DDPF) that allows peers/network elements to exchange data traffic between meshed base stations where WiMAX doesn't allow this. The proposed solution is layer-3 and includes the design of a routing table for a mesh configuration of cell-site (radio base station) routers with a base station controller. Some changes are needed which will be explored throughout the paper promoted with figures to illustrate the idea.
\end{abstract}

Keywords: Base Station- to -Base Station Mesh, WiMAX Mesh Network, Data Path Function, GRE Tunnel, R6 Interface

\section{Introduction}

WiMAX (Worldwide Interoperability for Microwave Access) based on IEEE 802.16 standards is a Wireless Metropolitan Area Network (WMAN) which supports point to multi-point Broadband Wireless Access (BWA) (WiMAX ForumTM, 2009), Fig. 1 shows the WiMAX network architecture. The IEEE wireless standard has a range of up to 30 miles and offers wireless access at lower cost with higher bandwidth (WiMAX Forum, 2009). It can afford transmission speed of up to 75 megabits per second. Therefore, WiMAX is considered one of the most attractive technologies for last-mile BWA problem in metropolitan areas and underserved rural areas. Three options are popular today for design of wireless networks - Point-to-Point, Point-to- Multipoint and Mesh topologies. WiMAX is most often associated with the point-to-multipoint (PMP) topology. As an example, Fig. 2 shows the base stations are configured in point-to-multipoint to the Access Service Network Gateway (ASN GW). In WiMAX for some requirements, the configuration of the Base Stations (BSs) is aimed to be as mesh (IEEE 802.16-2004, 2004). Mesh offers a combination of point-to-point and pointto-multipoint capability by having each of the base station able to communicate with other base station (IEEE Standard, 2015). This protects the base station element in the standard, besides the process flow across relevant interfaces. Some wireless technologies allow peer/network elements to negotiate by exchanging control signals but not data/bearer packets. The intension of this work is to have a function that allows peers/network elements to exchange data traffic where the wireless standard doesn't allow this.

A function is proposed in this work called distributed data path function (DDPF) that refers to a mesh mapping table (MMT) in order to guide/breakout the traffic. The mapping table describes the configuration of the network where the source, destination, data path identifications are recorded in it. The table allows single or multi-hop configurations. It can be fixed for fixed topology or it can be adapted and changed by the operator as part of the Operation and Maintenance (O \& M). An apparatus for example: a controller or a router or chip of a gateway or a module can implement the explained mechanism as part of its organization. The main goal of this work is to develop an effective method to mesh two base stations in WiMAX and to propose the necessary changes for this. The proposed solution is Layer-3 based solution that avoids modification to BS (stay compliant). The rest of 
the paper is organized as follows. In the next section we review the previous related work and highlight some recent papers. Another section describes the proposed method to mesh the base stations in WiMAX with the required tools followed by a section on the implementation guide to apply the proposed solution with layer-3 device and the required components to do the mesh. Finally, conclusions and the future research direction are given.

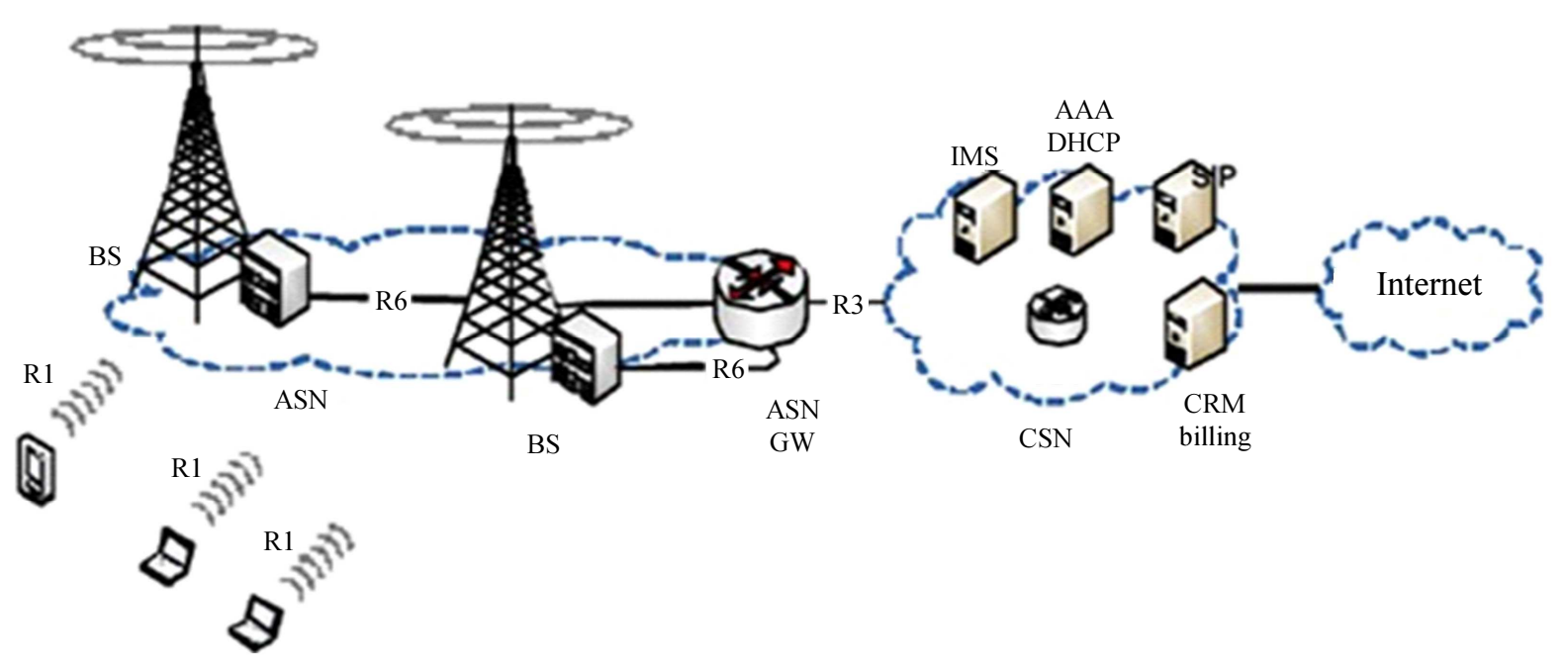

Fig. 1: WiMAX network architecture (WiMAX ForumTM, 2009)

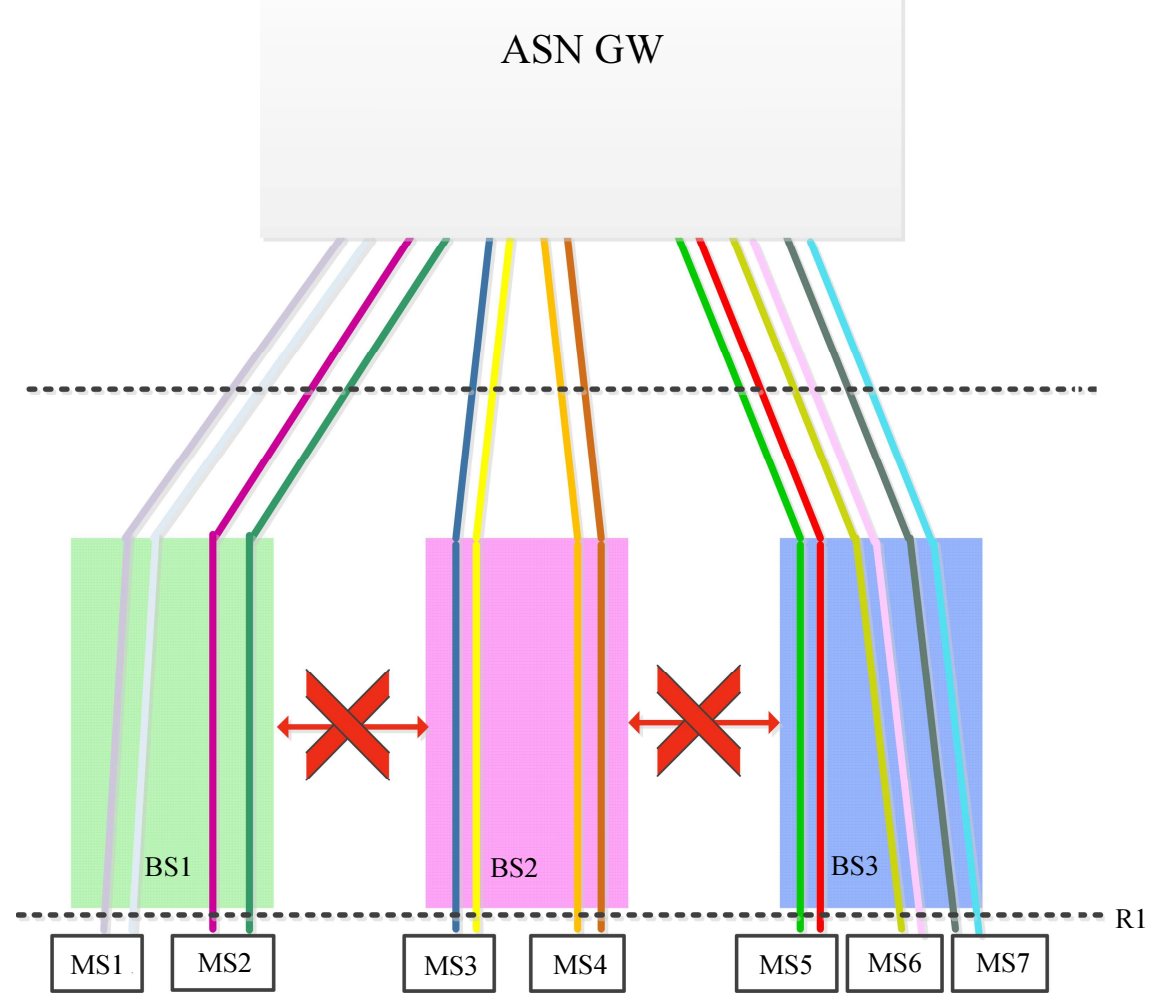

Fig. 2: The problem of exchanging data/bearer traffic in WiMAX networks, Note: R1, R6: WiMAX Interfaces; BS1, BS2, BS3: WiMAX Base Stations; MS1-MS7: Mobile Stations; ASN GW: Access Service Network Gateway Data/bearer traffic cannot traverse (the problem) 


\section{Literature Review}

Amin et al. (2008) presented an integrated routing and link scheduling approach to enable persistent communication of fast moving ground vehicles over a mesh-mode Mobile WiMAX tactical backbone network. They proposed to connect the base stations to the core network via satellite links in mesh mode. The mesh routing agent on each base station mesh radio implements a proposed Mobility-Aware Intra-Gateway Routing (MAIGR) protocol. Mobile WiMAX is the main concern of their work while our solution is targeting fixed WiMAX topology. Authors from Nokia Corporation in (US 2009/0073995 A1, 2009) proposed traffic breakout for Internet service domain traffic and operator's service domain traffic across R3 not R6 as proposed in this paper. It needs a permission from authentication, authorization and accounting (AAA) to allow local breakout. Breakout is only allowed in home agent and never in ASN GW. Address and port terminal is identified by either or combination of HoA, MS MAC (L2), data path ID, subscription ID, Network Access Identifier. The proposed switch in their work has a decision function to check the traffic type and forwards it correctly. Authors from Nortel Networks in (US 2006/0083186 A1, 2006) tried to improve quality of service over meshed backhaul facilities in a wireless network by providing redundant links between access points and limiting knowledge of these links locally to the access point. They proposed to advertise the aggregate link capacity globally to allow the aggregation of links to be treated as a single link for routing on the network of meshed access points. Xie and Wu (2008) proposed a method for negotiating interface data path establishment including: making a decision, by target ASN GW, on whether to establish an R4 data path or a redirect R3 data path and sending a corresponding request to the Anchor ASN GW according to the decision; and determining a negotiation result, by the Anchor ASN GW, according to the corresponding request from the target ASN GW, or the corresponding request from the target ASN GW and a local decision and returning a reply to the target ASN GW according to the negotiation result. In this way, the R3 data path is redirected if either the Target ASN GW or the Anchor ASN $\mathrm{GW}$ determines to redirect the R3 data path; and an R4 data path is established only if both the Target ASN GW and the Anchor ASN GW determine to establish the R4 data path.

Wolfe and Schnack (2006) proposed a method for providing radio communication coverage in a given area by implementing a mesh of Radio Base Stations (RBSs). Each RBS in the mesh includes a mobile interface for communicating with, one or more mobile terminals operating in its coverage area and a wireless backhaul interface for communicating with other RBSs in the mesh. At least one RBS in the mesh uses its backhaul interface to communicate with a mesh attachment point (MAP), which provides a communications link to a Base Station Controller (BSC). In Shi et al. paper (2018), the concept of local multicast was proposed to measure interference and solve hidden channel problem in multicast communication. Basing on the concept, they proposed a channel assignment algorithm considering the interference of Local Multicast and Forwarding Weight of each node (LMFW). The algorithm fully considers partially overlapped channels and orthogonal channels to improve the network performance. Li et al. (2012) in their study aims to analyze delay and throughput properties of an IEEE 802.16 mesh network for evaluating the performance of various real-time applications. They establish an analytical model to calculate delay and throughput of IEEE 802.16 distributed scheduling schemes. The proposed model helps to investigate how delay and throughput vary in terms of network parameters in order to optimize the system design via proper parameter configuration. The objective of the present paper is to find a solution that keeps the base station away from any change when meshing, so any changes to enable routing in the BS-toBS mesh network would have to be implemented in Layer-3 devices. Cell-site router is proposed to implement the process explained throughout this paper.

\section{Methodology}

This section explores the main elements in WiMAX standard that can help meshing base stations without changing the standard. Layer-3 solution is the recommended one to reach the target.

\section{Data Path Function (DPF)}

Data Path Function (DPF) is one of many functions defined in WiMAX standard as depicted in Fig. 3. One of DPF responsibility is Generic Routing Encapsulation GRE-tunneling of the backhaul traffic from the ASN GW in downlink towards the BSs and then the Mobile Stations (MNs)/wireless devices. This is done based on the source and destination addresses in the IP header and the key values in the GRE header. GRE breakout rules, e.g., detecting and switching rules, may be listed in a proposed Mesh Mapping Table (MMT).

DPF manages the setup of the bearer plane between two peers. This may include setup of any tunnels and/or additional functionality that may be required for handling the bearer plane. Each DPF is responsible for instantiating and managing data bearer between it and another data path function and for selecting the payload traversing the established data bearer. It is used to setup the bearer plane between base-stations or between other entities such as gateways or between gateways and basestations. As per WiMAX standard, UDP/IP shall be used as the transport protocol for communication between 
peer DPFs. The peer DPF at each end is addressed by the ID of the Network Component which hosts the data path (e.g., BSID), in combination with its Function Type which is part of the WiMAX NWG Message Header shown in Fig. 4. The highlighted fields in Fig. 4 are the focus of our approach.

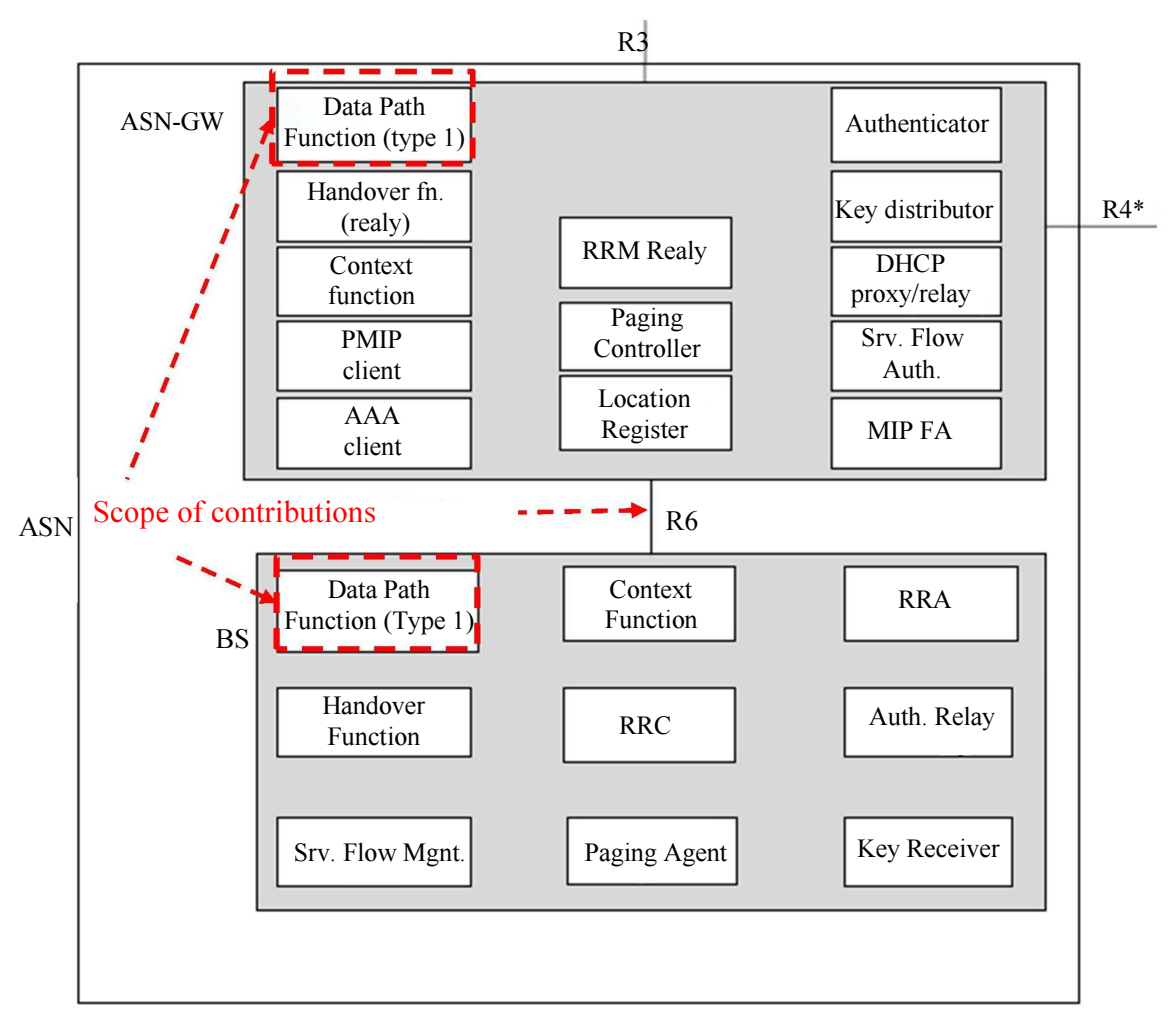

Fig. 3: Functional View of ASN GW and BS (IEEE Standard, 2015)

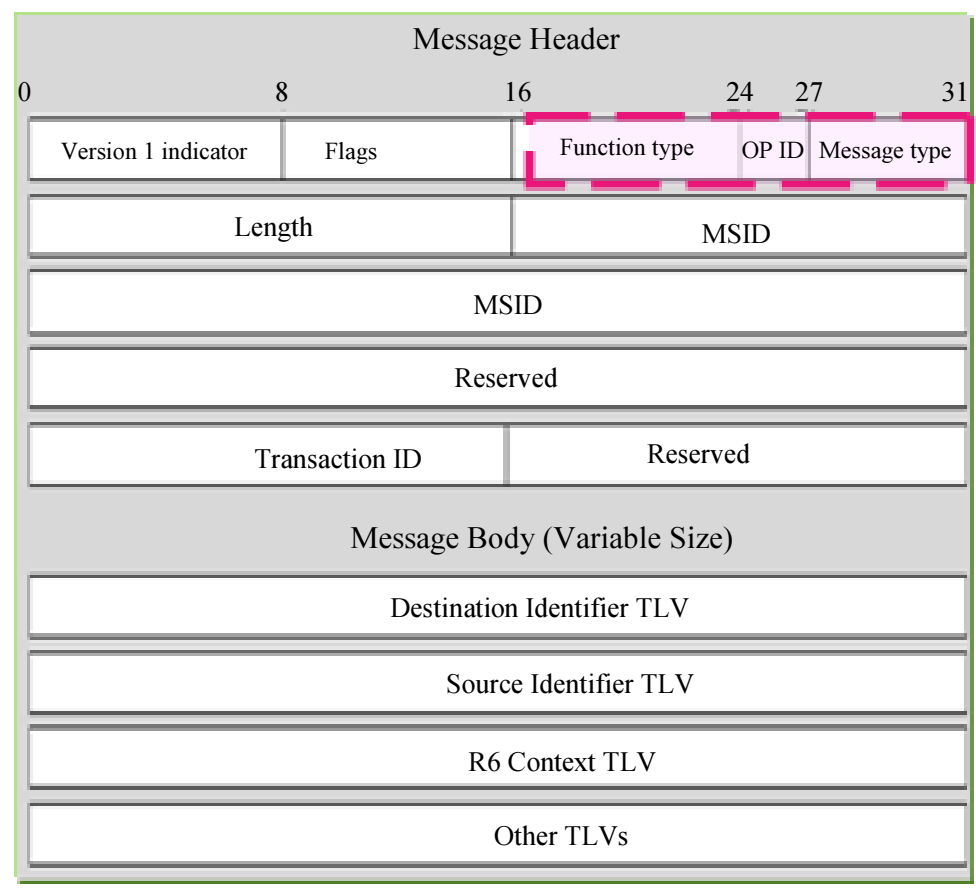

Fig. 4: WiMAX MAC Message Format (IEEE Standard, 2015) 
Many technologies can be used for tunneling such as Generic Route Encapsulation (GRE), Multiprotocol Label Switching (MPLS) and 802.1Q VLANs. Granularity of tunnels is defined and handled by the data path function. Generic Routing Encapsulation (GRE) is a tunneling protocol developed by Cisco that can encapsulate a wide variety of network layer protocol packet types inside IP tunnels, creating a virtual point-to-point link to Cisco routers at remote points over an IP internetwork. After classifying and mapping the IP packets at the DPF at one end, it will be prepared to be sending to the DPF at the other end. The user payload shall be encapsulated/tunneled using GRE protocol (as per the standard) with an outer envelope having the addressing information that enables the intervening L2/L3 network to deliver the packet to the appropriate physical implementation.

In WiMAX, there are 14 function types defined in the standard (IEEE Standard, 2015): 1. (QoS) 2. (HO
Control) 3. (Data Path Control) - with 14 messages 4. (Context Transfer) 5. (R3 Mobility) 6. (Paging) 7. (RRM) 8. (Authentication Relay) 9. (MS State) 10. IM Operations 11. Accounting 12. R4R6R8 Capability. Our intention in this paper is function type 3 which is the data path control with 14 messages shown in Table 1. In the following paragraphs, an illustration is made referring to Fig. 5 on how to mesh base station WiMAX. There should be a function that emulates the features of DPF, for this reason a distributed data path function (DDPF) has been proposed. DDPF will receive the source traffic which consists of the user payload and chain of headers preceding it. The headers can be encapsulation header or transport IP header. The DDPF scans the headers to see their types and the fields to be changed in each type. If the traffic is IP traffic this means that the relevant fields of IP header exists.

Table 1: WiMAX Data Path Control Messages: (Function Type 3) (IEEE Standard, 2015)

\begin{tabular}{|c|c|c|c|c|}
\hline Message Layout & Message & OP ID & Msg type & Function type \\
\hline \multirow[t]{14}{*}{3 (Data Path Control) } & 1 & 001 & Path_Dereg_Req & Table 4-35, Table 4-51, Table 4-75 \\
\hline & 2 & 010 & Path_Dereg Rsp & Table $4-76$ \\
\hline & 3 & 011 & Path_Dereg_Ack & $\begin{array}{l}\text { This message does not contain any } \\
\text { TLVs, so there is no message layout }\end{array}$ \\
\hline & 4 & 001 & Path_Modification_Req & Table $4-72$ \\
\hline & 5 & 010 & Path_Modification_Rsp & Table $4-73$ \\
\hline & 6 & 011 & Path_Modification_Ack & Table 4-74 \\
\hline & 7 & 001 & Path_Prereg_Req & Table $4-87$ \\
\hline & 8 & 010 & Path_Prereg_Rsp & Table 4-88 \\
\hline & 9 & 011 & Path_Prereg_Ack & Table 4-89 \\
\hline & 10 & 001 & Path_Reg_Req & Table 4-94 \\
\hline & 11 & 010 & Path_Reg_Rsp & Table 4-95 \\
\hline & 12 & 011 & Path_Reg_Ack & Table 4-96 \\
\hline & 13 & 100 & IM_Exit_State_Ind & Table 4-175 \\
\hline & 14 & 011 & IM_Exit_State_Ind_Ack & Table 4-176 \\
\hline
\end{tabular}

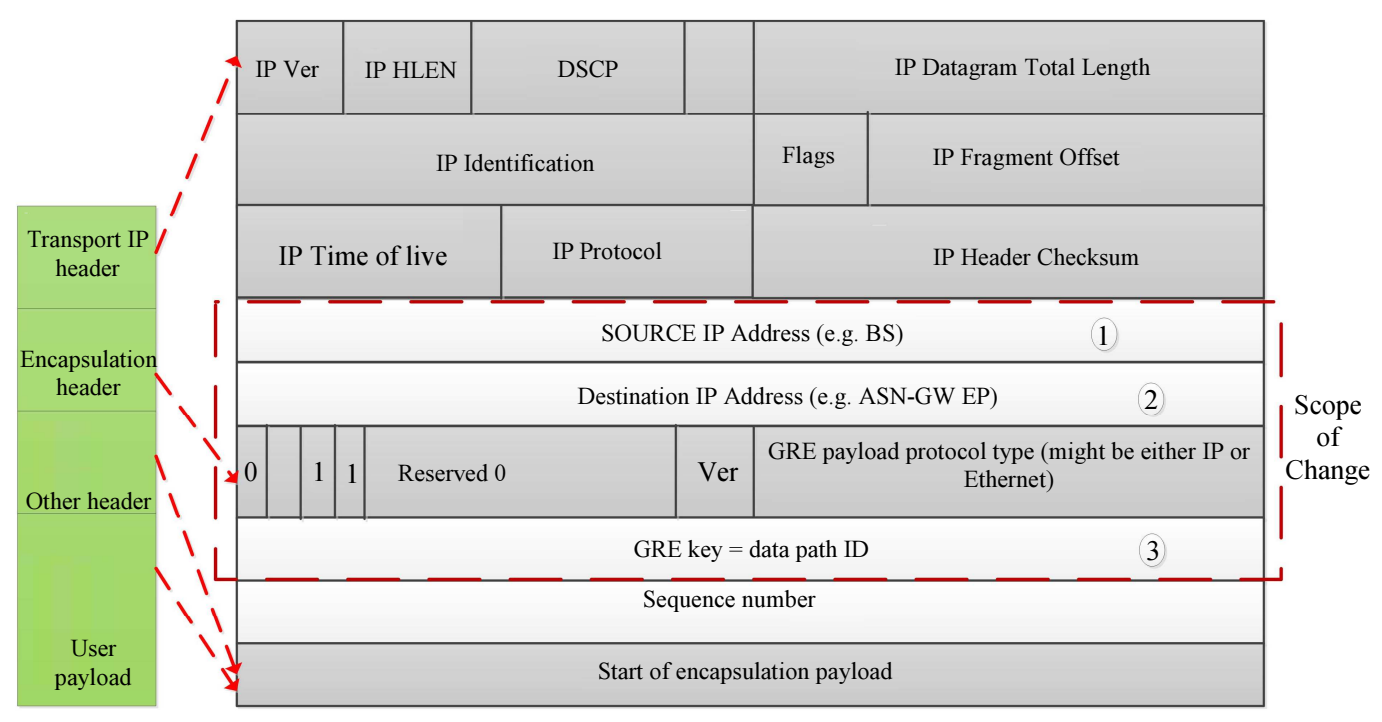

Fig. 5: IP and Encapsulation headers details 
If the traffic is tunneled traffic, the encapsulation header is expected to be found following the IP header. The detailed fields of the two headers are shown in Fig. 5.

In order to guide the traffic to the intended direction within the configured topology, the DDPF has to change two fields in the IP header (1 and 2 in Fig. 5) if the traffic is not capsulated. For encapsulated traffic, three fields (1,2 and 3 in Fig. 5) will be changed by DDPF. The source address (1 in Fig. 5) of the IP header should refer to the source IP address from where the traffic comes. The destination IP address (2 in Fig. 5) of the IP header should refer to the targeted destination where the traffic will go. The path for the traffic to follow is represented by data path identification (3 in Fig. 5).

\section{Mesh Mapping Table (MMT)}

In order for the DDPF to get the correct values of the fields to amend, a proposed mesh mapping table (MMT) is introduced. The MMT explains the topology configuration; it can handle single or multi-hop scenarios; it can be part of Operation and Maintenance $(\mathrm{O} \& \mathrm{M})$.The mapping table will guide the traffic to the requested destinations (for example, mesh topology). The steps that have been proposed and followed in this paper for meshing is illustrated in Fig. 6. This computer program module product can be stored in a computer program readable medium. According to one aspect of this paper, the backhaul traffic consists of user plane traffic and the control plane traffic. The user plane traffic includes the encapsulation user's payload-GRE traffic. The control plane traffic includes WiMAX control messages and User Datagram Protocol/Internet Protocol (UDP/IP). Figure 7 shows current WiMAX point-to-point traffic dissemination. Figure 8 depicts an overview of GRE traffic breakout and distributing it to many devices such as Base Stations (BSs) and Cell-Site Routers (CSRs). The contributed Distributed Data Path Function (DDPF), the Mesh Mapping Table (MMT) and many GREs are shown in this figure.

(1) Each network element in the topology should have (if not, obtain from DHCP server) an address (IP or MAC) for routing purposes.

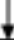

(2) As per WiMAX standard, the DPF will tunnel the service flows in GRE tunnel, each service flow should have key value (data path Identification).

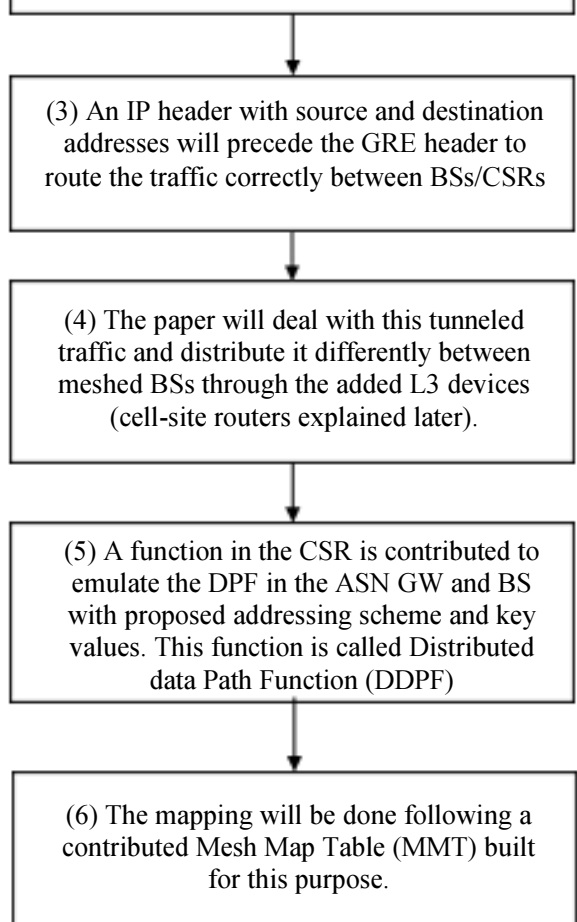

Fig. 6: Flow chart of the proposed steps for mesh process 
Table 2: Example mesh mapping table (MMT) for the topology in Fig. 8

\begin{tabular}{|c|c|c|c|c|c|}
\hline \multirow[b]{2}{*}{ DDPF (source) } & \multirow[b]{2}{*}{ Action } & \multicolumn{3}{|l|}{ Destination } & \multirow{2}{*}{$\begin{array}{l}\text { End User } \\
\text { (Connection Identification) }\end{array}$} \\
\hline & & Network element & Apparatus & Tunnel number & \\
\hline \multirow[t]{9}{*}{1} & Forward to & 1 & & 1 & 1 \\
\hline & & & & & 2 \\
\hline & & & & & 3 \\
\hline & & & & & 4 \\
\hline & & & & & 5 \\
\hline & Route to & & 2 & 2 & 6 \\
\hline & & & & & 7 \\
\hline & & & & & 8 \\
\hline & & & & & 4 \\
\hline \multirow[t]{5}{*}{2} & Forward to & 2 & & 4 & 5 \\
\hline & & & & & 6 \\
\hline & & & & & 7 \\
\hline & Route to & & 3 & 3 & 8 \\
\hline & & & & & 7 \\
\hline 3 & Forward to & 3 & NONE & 5 & 8 \\
\hline
\end{tabular}

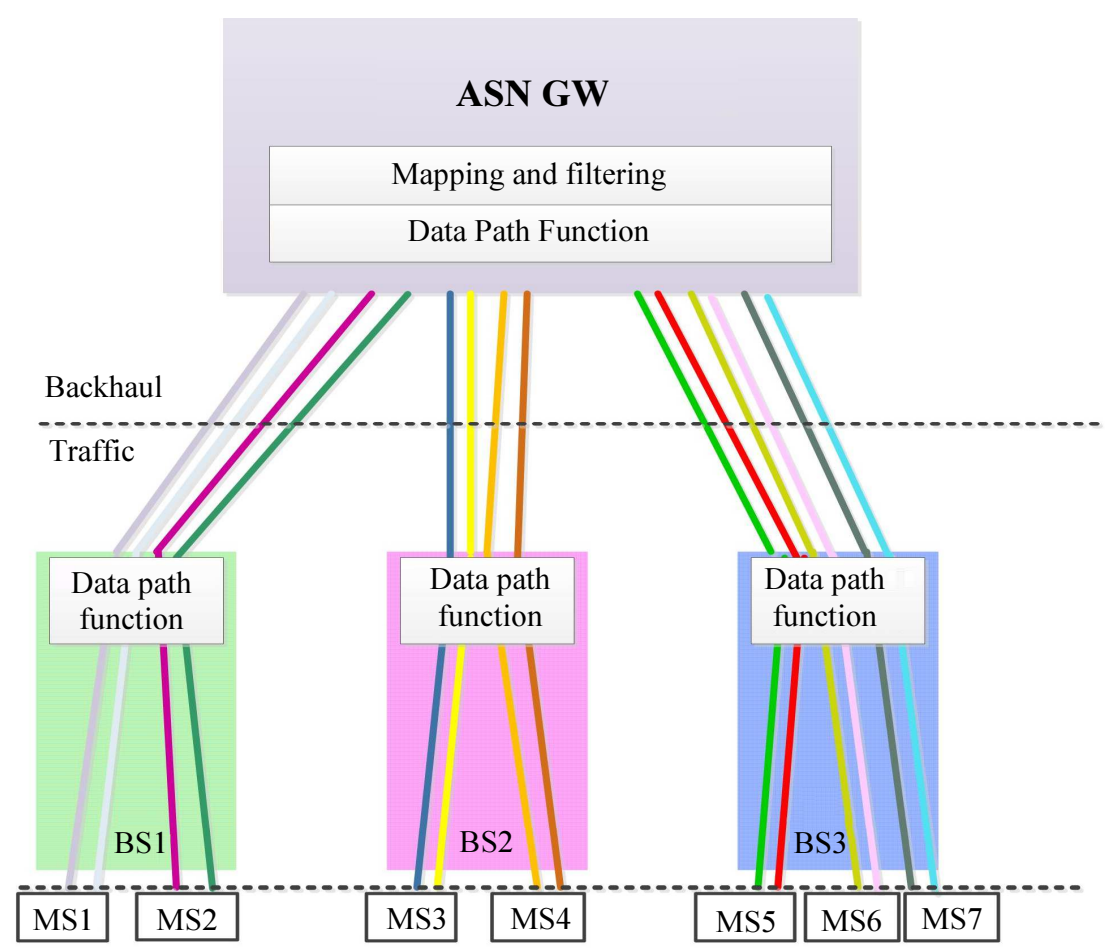

R1, R6: WiMAX interface; BS1, BS2, BS3: WiMAX base station;

MS1-MS7: Mobile station; ASN GW: Access Service Network Gatway

Fig. 7: ASN GW-to-BSs traffic dissemination in current point-to-point scenario

Traffic mapping/dissemination is done referring to the MMT which can be broadcasted/multicasted to all neighboring nodes (WiMAX Forum, 2009). The values of the source, destination and key/tag/connection identification values in Table 1 are obtained by the DDPF from the IP and encapsulation headers after decapsulation. They are recorded in the MMT for further reference such as routing. The MMT should be updated in case of mobility, handover, configuration change, classification rule change or any other reason that lead to topology change, it allows for single or multi-hop topology. For example if we have the topology shown in Fig. 8, where the source data traffic is to be distributed amongst network elements configured in any fashion. 


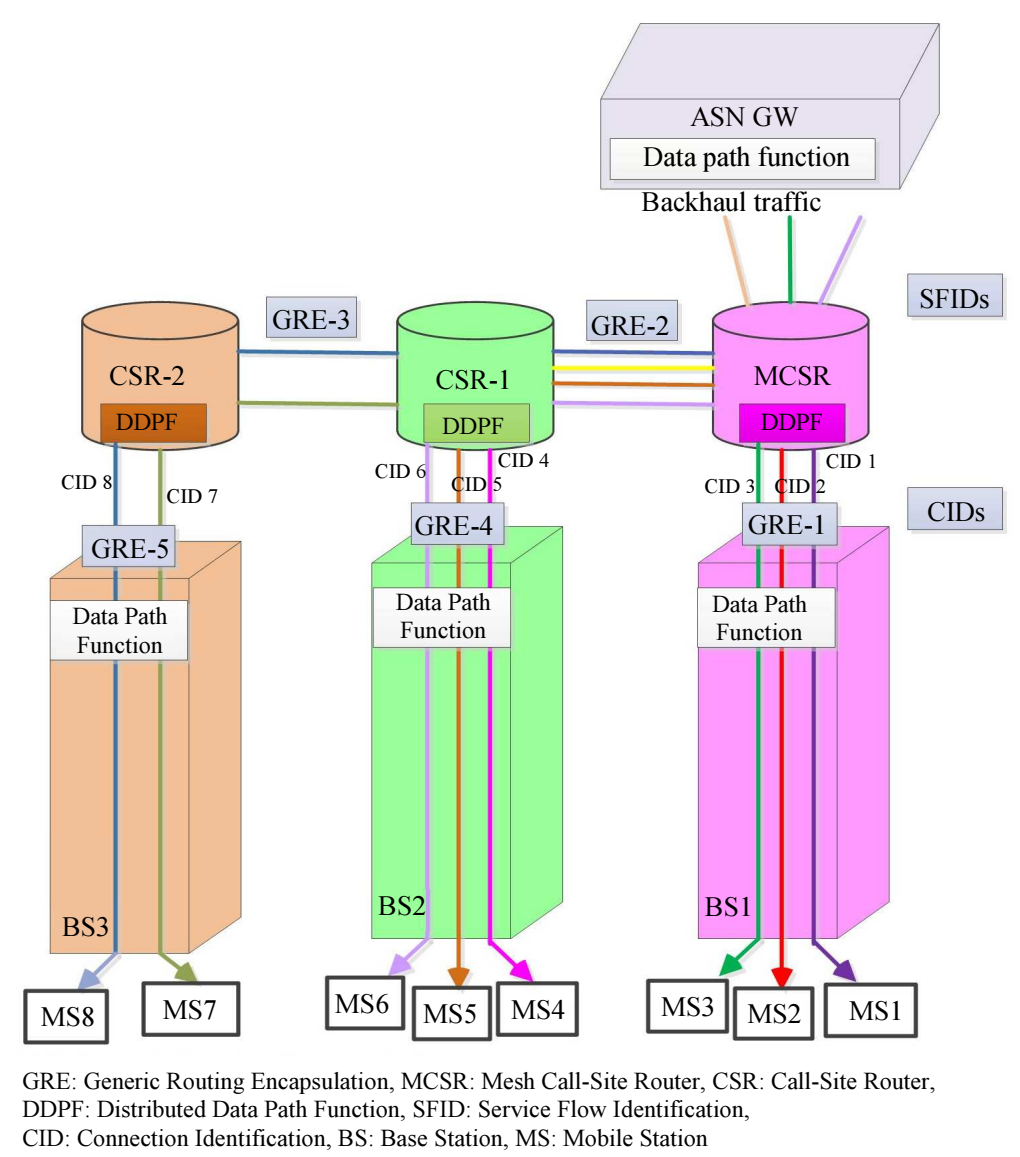

Fig. 8: ASN GW-to-BSs traffic dissemination in targeted mesh scenario

The topology map for this example is illustrated in Table 2. For example DDPF1 in the first apparatus will receive the source traffic (tunneled or not), it follows the steps explained in Fig. 6 for mapping/disseminating the traffic to two paths/tunnels, path/tunnel-1 towards network element- 1 then users 1,2 and 3 and path/tunnel-2 towards DDPF2 in another apparatus. Similarly, DDPF2 will map/disseminate the traffic to Path/Tunnel-3 and Path/Tunnel-4 then to network elements and DDPF in another apparatus towards end users and so on.

\section{Implementation and Discussion}

An apparatus may comprise means for receiving backhaul traffic from the ASN GW and means for routing the different traffic types to different paths or networks (Singh, 2019). The DDPF is part of the apparatus/device that maps/disseminates data/bearer traffic to predefined destinations recorded in a mapping table MMT.

This may include setup of any tunnels and/or additional functionality that may be required for handling the bearer plane. The intention of this paper is to find a method to distribute the backhaul traffic in between meshed BSs. This is done by breaking out the traffic, redistribute it and map it using a mapping table as explained in previous section. Cell-Site Router (CSR) a layer-3 device is proposed for this reason. Each base station is supposed to be attached to a CSR, accordingly, the interfaces and relevant functionality should be adapted. The network reference model for the paper shown in Fig. 10 comprises of the ASN GW and L3 devices (CSRs/MCSRs) each connected to a base station. The physical implementation of the link between the mesh cell site routers may use any appropriate technologies (such as wireless 802.3 bridge). This and the architecture of the system are out of the scope of this paper. There are set of messages for each wireless standard to set up, modify or remove the data path (HUNG-YU wei, 2013). In this paper, the flow of messages (WiMAX example) is changed to cater the required process of the proposed DDPF. The new flow of messages to/from the proposed DDPF is shown in Fig. 9. A GRE breakout can be provided in the cell-site routers to forward/route the received traffic as per the data path function.

In accordance with the description explained in this paper, an apparatus (CSR) is configured to receive backhaul traffic and to route the traffic to different paths or networks so as to provide a GRE breakout function or device. 


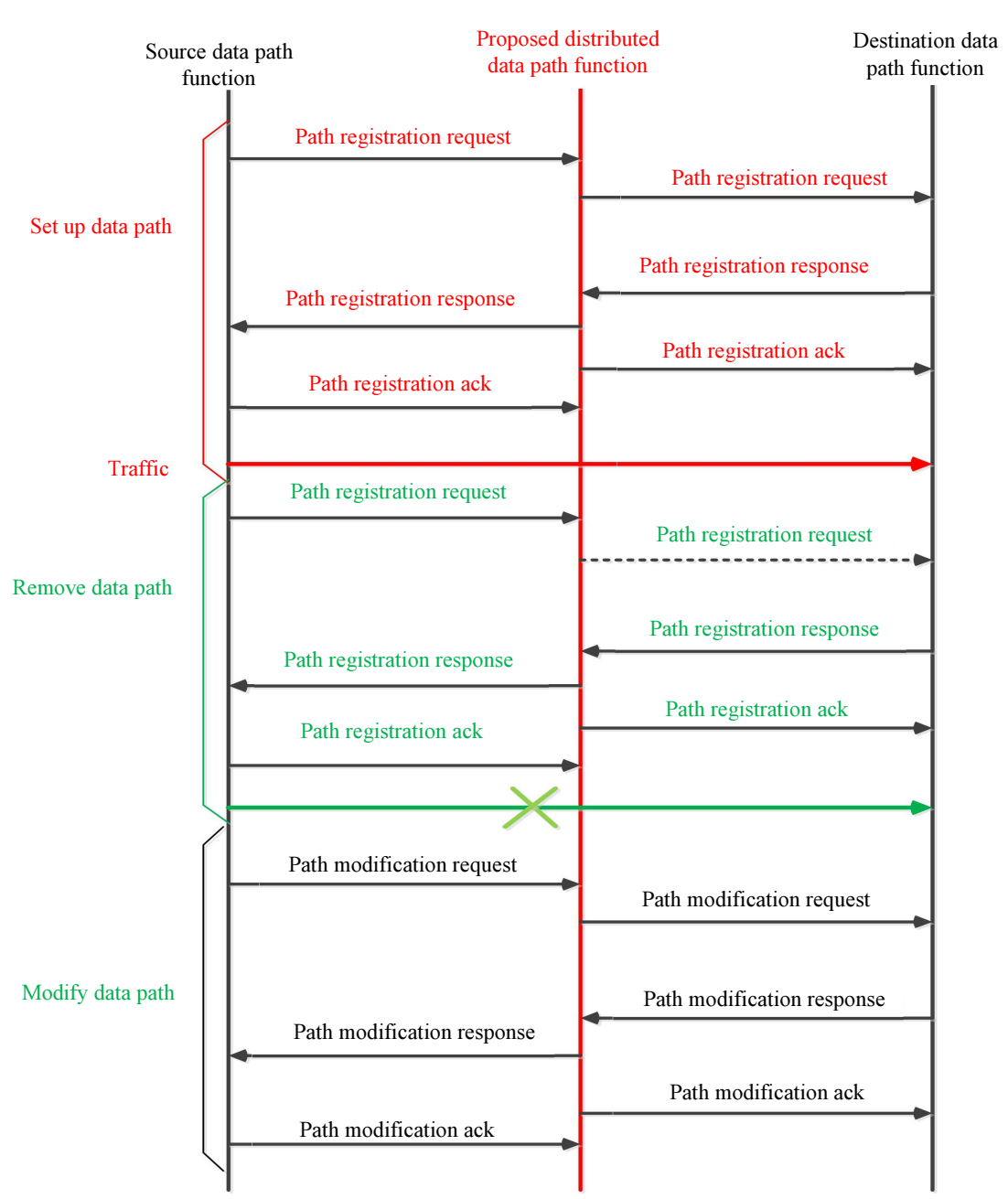

Fig. 9: Message flows to/from the proposed DDPF using WiMAX set of messages

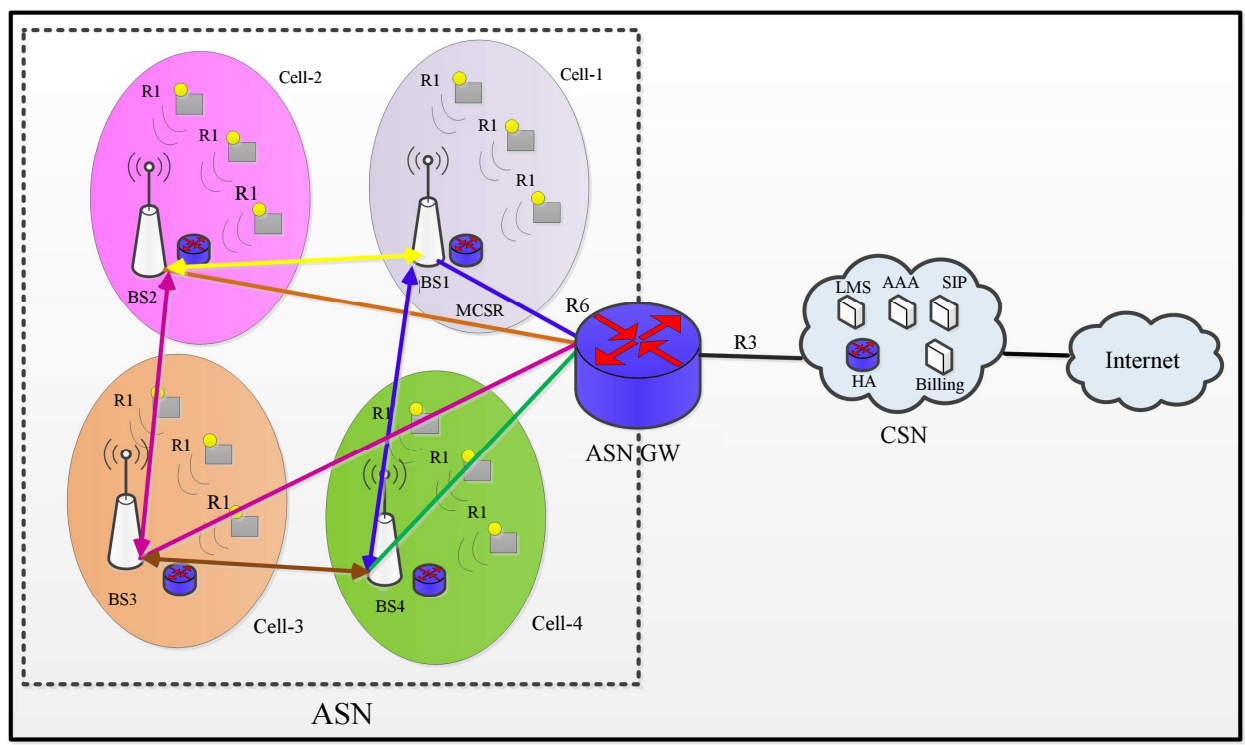

Fig. 10: Network reference model for the WiMAX BS-to-BS mesh with the proposed cell-site routers 
The apparatus may be a device, module or chip of a router or another device and/or may comprise at least one of a switch configured to switch incoming traffic to mesh base stations or another cell-site router, a data path function and a network address translation function or network address port translation function. The switch may be at least one of a layer 3 switch and a public or private switch and/or may be provided between the data path function or a gateway input and other traffic tunnel end point.

Figure 11 shows more details of the breakout process. Figure 12 illustrates the proposed apparatus with the contributed function DDPF and the mesh mapping table.

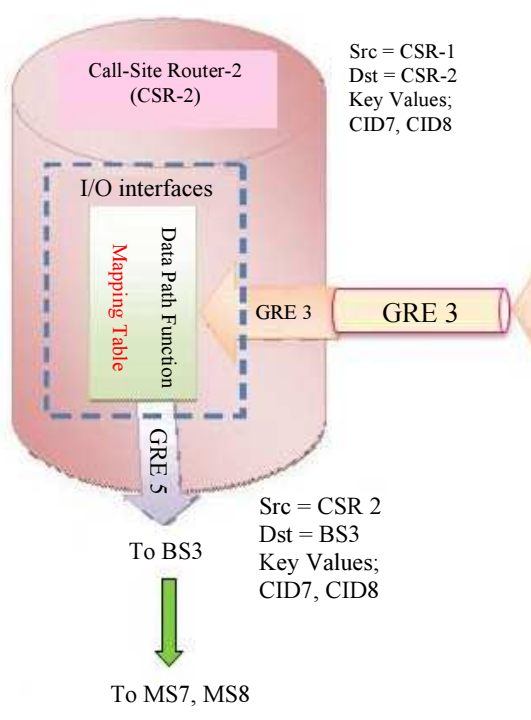

CSR-1, CSR-2, MCSR: Call-Site Routers BS1, BS2, BS3: Base Stations

GRE, GRE 1, GRE 2, GRE 3, GRS 4, GRE 5: Generic Routing Encapsulation Tunnels MS1-8: Mobile Stations, CID1-8; Connection identifiers

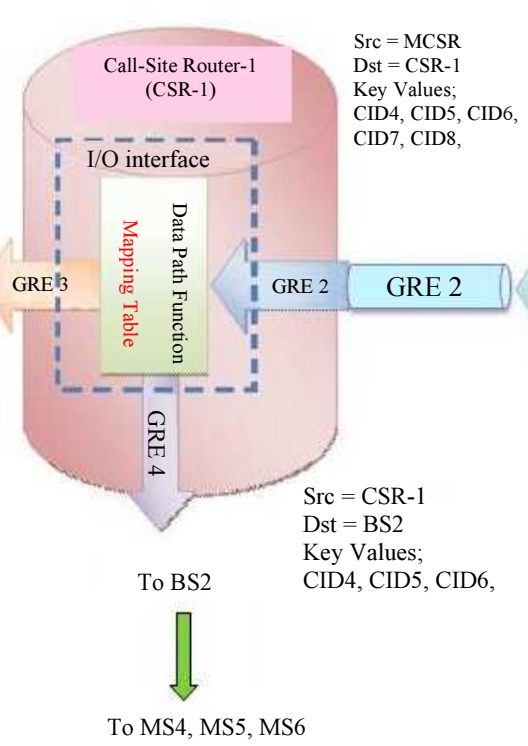

o MS4, MS5, MS6

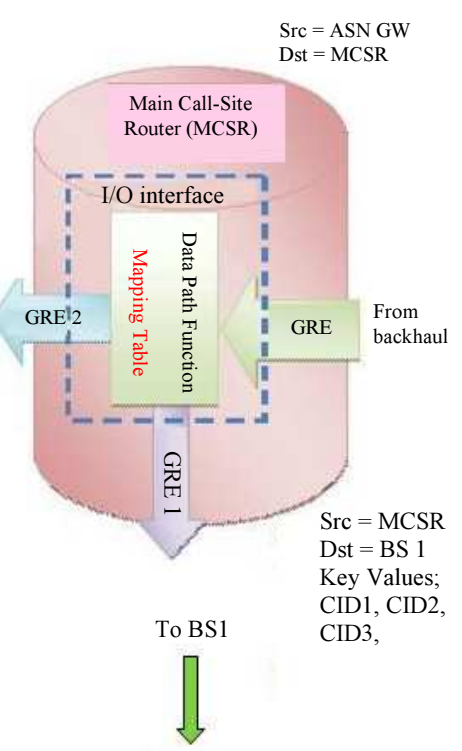

To MS1, MS2, MS3

Fig. 11: Overview of the process of GRE breakout of backhaul traffic and distributing it to many devices such as BSs and CSRs

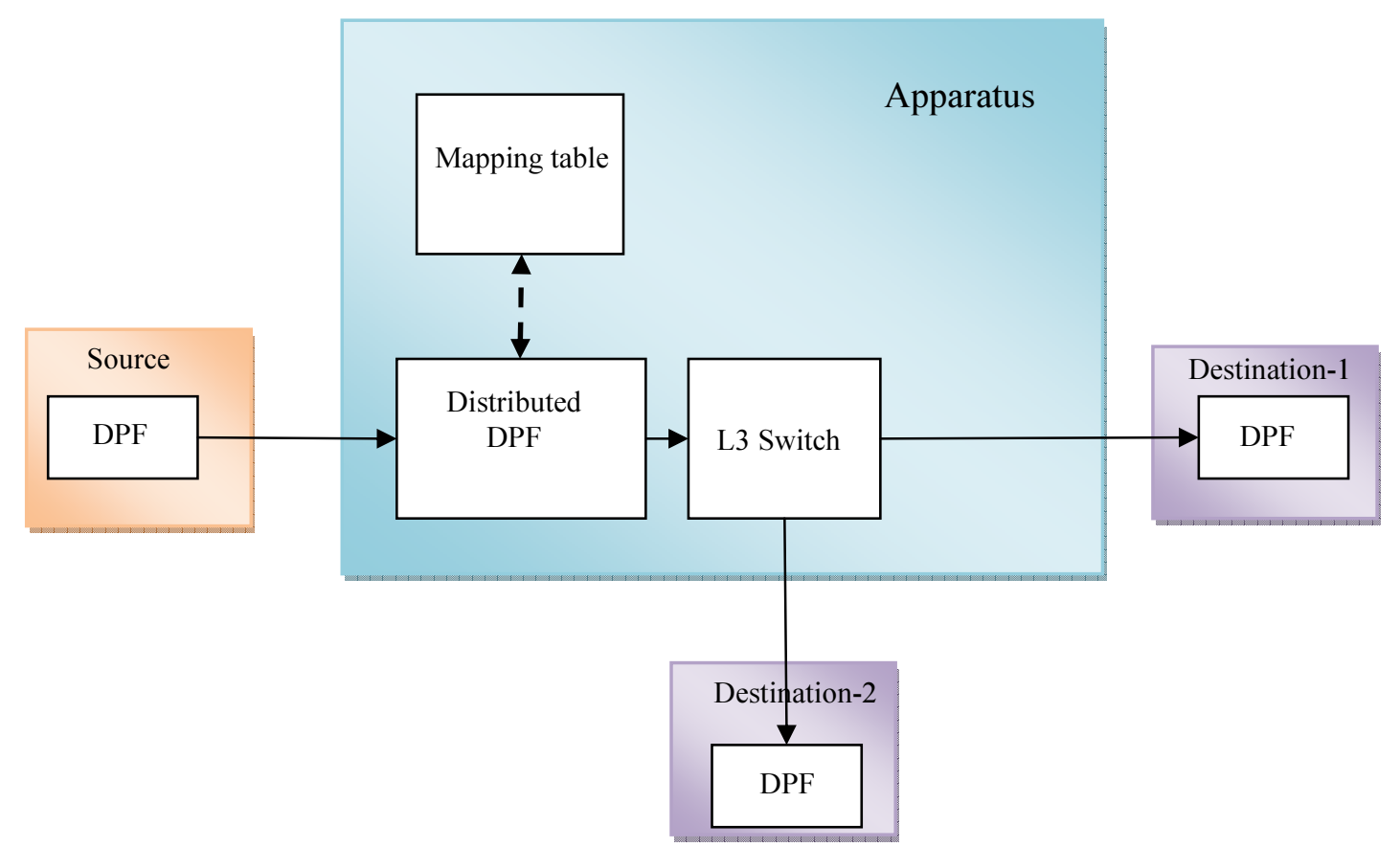

Fig. 12: Main Components of the proposed apparatus 


\section{Conclusion and Future work}

Although the description in this paper is in terms of a WiMAX network, the illustrated method is not limited to this application, but is generally applicable to any wireless network. Layer-3 solution to mesh base stations is introduced in this paper via cell-site router which will receive backhaul traffic and means for routing the traffic to different paths or networks, the backhaul traffic comprising data and control traffic tunneled/encapsulated and send from the ASN GW towards the base stations via this router.

The proposed and explained scheme in this paper is not restricted to the illustrated example architectures or configurations, but can be implemented using a variety of alternative architectures and configurations. Further work is needed on QoS, accounting, R3 mobility, or handover control.

A simulation can be built in order to get results that can be compared and benchmarked with a standard network.

\section{Author's Contributions}

All authors equally contributed in this work.

\section{Ethics}

This article is original and contains unpublished material. The corresponding author confirms that all of the other authors have read and approved the manuscript and no ethical issues involved.

\section{References}

Amin, R., K.C. Wang and P. Ramanathan, 2008. An integrated routing and scheduling approach for persistent vehicle communication in mobile WiMAX Mesh networks. Proceedings of the Milcom, Nov. 16-19, pp: 1 - 7.

HUNG-YU wei, 2013. Jarogniew Rykowski and Sudhir Dixit, "WIFI, WiMAX and LTE mult-hop Networks: Basic communication protocols and application areas, John Wiley and Sons, Inc.
IEEE 802.16-2004, 2004. IEEE Standard for Local and Metropolitan Area Networks- Part 16: Air Interface For Fixed Broadband Wireless Access Systems, IEEE Standard.

IEEE Standard, 2015. IEEE Standard for air interface for broadband wireless access systems, amendment 3: Multi-tier Networks, in IEEE 802.16q - 2015 (Amendment to IEEE standard 802.16-2012), pp: 1-79.

Li, Y., C. Wang and X. You 2012. Delay and throughput performance of IEEE 802.16 WiMax mesh networks. IET Communications, 6: 107-115. DOI: $10.1049 /$ iet-com.2010.1031

Shi, W., S. Wang, Z. Wang and E. Wang, 20018. An efficient channel assignment algorithm for multicast wireless mesh networks. AEU Int. J. Electronics Communications, Elsevier, 89: 62-69.

Singh, M., 2019. Node -to -node approaching in wireless mesh connectivity. Springer Singapore, ISBN-10: 978-981- 0674-7.

US 2006/0083186 A1, 2006. Nortel networks limited, "Method and Apparatus for improving Quality of Service over meshed backhaul facilities in a Wireless Network".

US 2009/0073995 A1, 2009. Nokia Corporation, "Devices and Methods for Local Breakout in a Gateway of an Access Service Network", Publication date: 19 March 2009.

WiMAX Forum, 2009. Network Architecture "stage 2: Architecture Tenets, Reference Model and Reference Points", Part 1, Release 1.0 Version 4, WMF-T32-002-R010v04 (February 03, 2009).

WiMAX ForumTM, 2009. Network architecture "Detailed Protocols and Procedures Base Specification" DRAFT-T33-001-R015v01-I Draft Specification (2009-06-03).

Wolfe C. and Schnack L., 2006. Radio access network with meshed radio base stations. US 6,996,086 B2.

Xie and $\mathrm{Wu}$, J., 2008 Method and system for negotiating interface data path establishment, US 2008/0104192 A1. 